OPEN ACCESS

Edited by:

Weiming Tang,

University of North Carolina at

Chapel Hill, United States

Reviewed by:

Huachun Zou,

Sun Yat-sen University, China

Weiming Zhu,

Centers for Disease Control and

Prevention $(C D C)$, United States

*Correspondence:

$\mathrm{NaHe}$

nhe@fudan.edu.cn

${ }^{\dagger}$ These authors have contributed equally to this work

Specialty section:

This article was submitted to Microbiome in Health and Disease,

a section of the journal

Frontiers in Cellular and Infection Microbiology

Received: 06 July 2021 Accepted: 04 October 2021 Published: 25 October 2021

Citation:

Dong $R$, Lin $H$, Chen $X$, Shi R, Yuan S, Li J, Zhu B, Xu X, Shen W, Wang $K$, Shu $X-O$, Ding D and He N (2021) Gut

Microbiota and Fecal Metabolites Associated With Neurocognitive Impairment in HIV-Infected Population. Front. Cell. Infect. Microbiol. 11:723840. doi: 10.3389/fcimb.2021.723840

\section{Gut Microbiota and Fecal Metabolites Associated With Neurocognitive Impairment in HIV-Infected Population}

\author{
Ruihua Dong ${ }^{1,2 \dagger}$, Haijiang $\mathrm{Lin}^{2,3 \dagger}$, Xiaoxiao Chen ${ }^{3}$, Ruizi Shi ${ }^{2}$, Shiying Yuan ${ }^{2}$, Jing $\mathrm{Li}^{2}$, \\ Bowen Zhu ${ }^{2}$, Xiaohui $\mathrm{Xu}^{2}$, Weiwei Shen ${ }^{3}$, Keran Wang ${ }^{2}$, Xiao-Ou Shu ${ }^{4}$, Ding Ding ${ }^{5}$ \\ and $\mathrm{Na} \mathrm{He}{ }^{2,6 *}$ \\ ${ }^{1}$ Department of Nutrition and Food Hygiene, School of Public Health, Fudan University, Shanghai, China, ${ }^{2}$ Department of \\ Epidemiology, School of Public Health and The Key Laboratory of Public Health Safety of Ministry of Education, Fudan \\ University, Shanghai, China, ${ }^{3}$ Department of Epidemiology, Taizhou City Center for Disease Control and Prevention, Taizhou, \\ China, ${ }^{4}$ Division of Epidemiology, Department of Medicine, Vanderbilt Epidemiology Center, Vanderbilt University Medical \\ Center, Nashville, TN, United States, 5 Institute of Neurology, Huashan Hospital, Fudan University, Shanghai, China, \\ 6 Shanghai Institute of Infectious Diseases and Biosecurity, Fudan University, Shanghai, China
}

Gut microbiota dysbiosis has been associated with many neurological diseases. However, how microbiota composition and metabolism relate to neurocognitive impairment (NCl) in HIV-infected individuals is largely unknown. In this study, a total of $102 \mathrm{HIV}$ infected participants were classified into two groups - those with $\mathrm{NCl}$ and those without-using the global deficit score (GDS). Fecal samples were collected from the participants for $16 \mathrm{~S}$ rRNA gene sequencing and untargeted metabolomics. The plasma level of 25 hydroxy-vitamin $\mathrm{D}(25(\mathrm{OH}) \mathrm{D})$ was also evaluated. Although $\alpha$-diversity and $\beta$ diversity were comparable, the HIV patients with $\mathrm{NCl}$ were significantly different from those without $\mathrm{NCl}$ in terms of abundance of several gut microbiota. The decreased abundance of butyrate-producing bacteria (BPB) and increased abundance of Klebsiella were related with $\mathrm{NCl}$ and carotid intima-media thickness (CIMT). Significant differences in fecal metabolites were also found between individuals with versus without $\mathrm{NCl}$, including increased bile acids and bioactive lipids, decreased vitamin D, terpenoids, and resolvin D1 in the $\mathrm{NCl}$ group. Furthermore, the perturbed metabolic profile was closely related to BPB and Klebsiella. In addition, a low level of vitamin D was associated with $\mathrm{NCl}$ and CIMT. Both fecal and plasma vitamin D were positively correlated with BPB. Our results show that BPB and Klebsiella and the associated metabolites are associated with $\mathrm{NCl}$ in people with HIV. In addition, vitamin D, both in feces and blood, was associated with $\mathrm{NCl}$ and $\mathrm{BPB}$, suggesting a protective effect of vitamin $\mathrm{D}$ on $\mathrm{NCl}$.

Keywords: HIV, neurocognitive impairment (NCI), gut microbiota, butyrate-producing bacteria, metabolomics, vitamin D 


\section{INTRODUCTION}

HIV is neurovirulent and frequently causes brain impairment (Williams et al., 2020). Therefore, HIV-infected individuals often experience a neurocognitive impairment (NCI), referred to as $\mathrm{HIV}$-associated neurocognitive disorders (HAND) (Heaton et al., 2010). The CNS HIV Antiretroviral Therapy Effects Research study has reported a prevalence of 35 and $50 \%$ of older adults living with HIV who experience mild to moderate cognitive impairment (Ding et al., 2017a; Ding et al., 2017b). The possible reasons for the persistent prevalence of NCI include accelerated aging of HIV population, direct damage from the virus, and neurotoxicity of specific antiretroviral drugs (Qiao et al., 2019). However, the pathogenesis of NCI in HIV-infected individuals still remains incompletely understood.

Gut microbiota dysbiosis is common in HIV-infected individuals, including decreased diversity and alterations in gut microbiome composition compared with healthy individuals (Ashuro et al., 2020). In HIV-infected individuals, loss of intestinal barrier integrity, an increased plasma level of microbiota products, and systemic inflammation have been correlated with NCI (Zhang et al., 2019). Furthermore, one investigation found that the HAND group presented a significantly lower $\alpha$-diversity compared with the non-HAND group, using the $16 \mathrm{~S}$ rRNA gene sequencing approach, suggesting a potential relationship between the gut microbiota and HAND (Zhang et al., 2019). Therefore, gut microbiota dysbiosis may play a role in the pathogenesis of NCI.

Of note is that non-targeted fecal metabolomics studies have been used in understanding metabolites associated with gut microbiota alteration in complex disease development (Luan et al., 2019) — for example, a study found distinct changes in the fecal metabolites of depressive rats, and the gut microbiota was altered in association with fecal metabolites (Yu et al., 2017). Nevertheless, no study has been conducted using integrative analysis of fecal metabolome and microbiome to understand the pathogenesis of NCI.

To explore the role of gut microbiota and the underlying metabolic mechanisms in the development of NCI among HIVinfected individuals, we applied 16S rRNA gene sequencing and non-targeted fecal metabolomics to compare gut microbiota and fecal metabolite differences among HIV-infected individuals with and without NCI.

\section{MATERIALS AND METHODS}

\section{Study Settings and Design}

The study participants were recruited from the Comparative HIV and Aging Research in Taizhou (CHART). The detail of the prospective cohort has been described previously (Ding et al., 2020). Between February and December 2017, 1,770 HIVpositive adults and 3,350 HIV-negative controls were enrolled from Taizhou Prefecture of Zhejiang Province, Eastern China. The samples of this study came from one of the substudies under the CHART cohort. The substudy included HIV-infected patients if they were between 40 and 80 years, able to communicate in Mandarin and literate, and had no serious hearing or eye problem. The included participants further took a comprehensive battery of neuropsychological (NP) test (supplementary materials) by certified professionals. The global cognitive score was defined by the global deficit scores (GDS) dichotomized as impaired (GDS $\geq 0.5$ ) or unimpaired (GDS < 0.5) (Cysique et al., 2007). In total, $385 \mathrm{HIV}$-infected patients and 411 controls completed the NP tests. Among them, $122 \mathrm{HIV}$ infected patients were identified with NCI, and 122 controls (HIV-infected patients without NCI) were randomly selected, with comparable age and gender. In 2019, we invited these participants to take the NP test again and provide their fecal samples. Then, $88 \mathrm{HIV}$-infected patients with NCI and 47 controls agreed to participate in this study and completed the NP test. After excluding the participants without fecal samples, a total of $67 \mathrm{HIV}$-infected persons with NCI and $35 \mathrm{HIV}$-infected persons without NCI were included in the present analysis (Supplementary Tables S2 and S3) The Ethics Committee of the School of Public Health at Fudan University approved this study. All individuals gave informed consent at enrollment.

\section{Data Collection}

For the baseline survey, all cohort participants were administered with a questionnaire interview to collect basic demographics including age, sex, education, lifestyle, etc. Physical examinations of height, weight, and blood pressures (BP) were carried out. BP was measured twice, and the average of the two readings were recorded. Body mass index (BMI) was calculated as body weight divided by the height squared $\left(\mathrm{kg} / \mathrm{m}^{2}\right)$ of the participant. Hypertension was defined as systolic BP $\geq 140 \mathrm{mmHg}$ or diastolic $\mathrm{BP} \geq 90 \mathrm{mmHg}$. They also received comprehensive physical and biochemical examinations and B-mode ultrasonic and electrocardiographic examinations (Qiao et al., 2019). The most recent CD4 $\mathrm{T}$ cell count was extracted from the National HIV/AIDS Comprehensive.

Neurocognitive performances were assessed using the NP test (Supplementary Materials) by certified professionals. The global cognitive score was defined by the GDS dichotomized as impaired (GDS $\geq 0.5$ ) or unimpaired (GDS <0.5) (Cysique et al., 2007). Depressive symptoms were measured by the 10 item version of Zung Self-Rating Depression Scale (Zung, 1973). The insomnia symptoms were measured based on Jenkins's fouritem sleep questionnaire (Jenkins et al., 1988). Frailty phenotype was assessed using the Fried criteria, with the exception of physical activity, for which we used the proxy described by Onen et al. (Ding et al., 2017a; Ding et al., 2017b).

\section{$16 S$ rRNA Sequencing and Measurement of Fecal Metabolomics}

We collected fecal samples from 102 participants of this study. The fecal samples were collected in a feces container and stored at $-80^{\circ} \mathrm{C}$. Total genomic DNA was isolated using DNA Extraction Kit (Qiagen, Düsseldorf, Germany) as per the instructions of the manufacturer. Details of the 16S rRNA sequencing and the quantitative measurement of fecal 
metabolomics as well as relevant data analysis are available in the Supplementary Materials.

\section{Hydroxy-Vitamin D [25(OH)D] Measurements}

The plasma samples were refrigerated immediately after phlebotomy and centrifuged and frozen in a central laboratory within $2 \mathrm{~h}$. Levels of 25(OH)D were measured using enzyme-linked immunosorbent assays and categorized as normal $(30 \mathrm{ng} / \mathrm{ml})$, insufficient (20 to $29 \mathrm{ng} / \mathrm{ml})$, and deficient $(<20 \mathrm{ng} / \mathrm{ml}$ ) (Manion et al., 2017).

\section{Statistical Analyses}

The demographic factors and clinical parameters were compared between the two groups. Continuous variables were presented as median (interquartile range) or mean (standard deviation) based on whether the data were normally distributed. Categorical variables were summarized as numbers and percentages (\%).

The $\alpha$-diversity indices, including Chao, Shannon, and Simpson, were calculated (Sun et al., 2016). Weighted and unweighted UniFrac distances were used to measure $\beta$ diversity (Ji et al., 2018). Differences in characteristics and diversity indices between groups were compared by Student's $t$-test (continuous variables, normal distribution), the MannWhitney $U$-test (continuous variables, skewed distribution), or chi-square test (categorical variables). Differences in the relative abundances of gut microbiota at the genus level between groups were assessed using a generalized linear model with sex, age, CD4 count, sexual preference, and BMI, included as co-variates. We further identified the differentially abundant taxa in the NCI and non-NCI groups by linear discriminant analysis effect size (LEfSe) (Li et al., 2019).

We performed orthogonal projection to latent structurediscriminant analysis (OPLS-DA) to examine the overall microbial metabolite distribution between the two groups. The fit and predictability of the models obtained were determined by the $R^{2} Y$ and $Q^{2}$ values, respectively. In the OPLS-DA model, we generated the variable importance plot (VIP) to select potential biomarkers. Depending on the distribution of the data, correlations between clinical parameters, fecal metabolites, 25(OH)D, and genera abundance were calculated by Spearman's rank test. Statistical significance was defined as two-sided and $P<0.05$. The $P$-values were adjusted to control the false discovery rate (FDR). OPLS-DA was performed with the software SIMCA-P+, version 14.0 (Wan et al., 2019). All other statistical analyses were performed in $\mathrm{R}$, version 3.6.2, and $\mathrm{R}$ packages (pheatmap, ggplot2, ggrepel, ropls, corrplot, and status) were applied in this work.

\section{RESULTS}

\section{Characteristics of the Study Participants}

Table 1 presents the demographics, clinical symptoms, and laboratory biomarkers of the 102 participants. There are no significant differences in the demographic measurements between the two groups. The HIV-infected participants in the NCI group had higher left and right carotid intima-media thickness (CIMT) than those in the non-NCI group. The NCI group also had a higher proportion of carotid plaques and cholesterol crystal than those in the non-NCI group (Table 1).

\section{Gut Microbiota Diversity Index and Gut Microbiota Composition}

An analysis of the $\alpha$-diversity showed that the Shannon, Simpson, and Chao indices were not significantly different between the NCI and non-NCI groups (Shannon index, $P=$ 0.95; Simpson index, $P=0.90$; and Chao index, $P=0.73$; Figures 1A-C). The $\beta$-diversity of gut microbiota was evaluated based on the unweighted UniFrac distance matrix $(P=0.082)$, and the weighted UniFrac distance matrix $(P=$ $0.459)$ showed no differences between the two groups in the fecal microbial communities (Figures 2A, B).

The species abundance of Spirochaetes and Epsilonbacteraeota was higher in the NCI group than in the non-NCI group at the phylum level. In addition, 56 genera showed significant differences between the two groups. The top 20 most abundant genera are presented in Supplementary Table S4. Only the differences in the abundance of Coprococcus_2, Treponema_2, Pseudomonas, and Thermopolyspora were significant between the two groups after FDR correction. LEfSe identified 36 discriminative features whose relative abundance varied significantly between the two groups. At the genera level, the microbiota of the non-NCI group was enriched with nine genera, while the NCI group was enriched with 27 genera (Figure 3). We conducted the following correlation analyses in the full cohort using the top 20 genera with the most abundant difference.

\section{Correlation Between the Abundance of Genera With Clinical Parameters}

Streptococcus $(P=0.014)$ and Treponema_2 $(P=0.020)$ were inversely correlated with CD4 count. Faecalibacterium, Ruminococcus_1, Coprococcus_2, and Ruminococcaceae _NK4A214_group were inversely correlated with the left CIMT, while Klebsiella was positively correlated with the left CIMT. Faecalibacterium was inversely correlated with right CIMT $(P=$ 0.018 ), while Klebsiella was positively correlated with the right CIMT $(P=0.018)$. However, we did not find a difference in FDR-corrected comparison (Supplementary Table S5). No significant relationship was found between the abundance of genera and other clinical parameters.

\section{Distinct Differences Between Fecal Metabolites in the NCI Group and Non- $\mathrm{NCl}$ Group}

The OPLS-DA model showed strong differences in the overall microbial metabolite distribution between the NCI and the nonNCI groups $\left(R^{2} Y=0.782, Q^{2}=0.215\right.$, Supplementary Figure S3). Twenty-four metabolites were identified as potential biomarkers. Supplementary Table S6 presents the corresponding retention time, $\mathrm{m} / \mathrm{z}$, and VIP values. 
TABLE 1 | Characteristics of the $\mathrm{NCl}$ and non-NCl groups $(n=102)$.

\begin{tabular}{|c|c|c|c|}
\hline Characteristics & $\mathrm{NCl}$ group $(n=67)$ & Non-NCI group $(n=35)$ & $P$-values \\
\hline \multicolumn{4}{|l|}{ Demographics } \\
\hline \multicolumn{4}{|l|}{ Sex, $n(\%)$} \\
\hline Male & $55(82.1)$ & $25(71.4)$ & 0.186 \\
\hline Female & $12(17.9)$ & $10(29.4)$ & \\
\hline Age, years, (mean \pm SD) & $54.6 \pm 9.7$ & $55.3 \pm 9.3$ & 0.708 \\
\hline $\mathrm{BMI}, \mathrm{kg} / \mathrm{m}^{2}($ mean $\pm \mathrm{SD})$ & $23.2 \pm 3.0$ & $22.9 \pm 2.6$ & 0.679 \\
\hline \multicolumn{4}{|l|}{ Education, $n(\%)$} \\
\hline$\leq$ Primary school & $49(70.0)$ & $20(49.0)$ & 0.297 \\
\hline Middle school & $13(19.4)$ & $11(31.4)$ & \\
\hline zHigh school & $5(7.5)$ & 4 (8.6) & \\
\hline \multicolumn{4}{|l|}{ Current smoker, $n(\%)$} \\
\hline Yes & 35 (52.2\%) & 13 (37.1\%) & 0.107 \\
\hline No & $32(47.8 \%)$ & 22 (62.9\%) & \\
\hline \multicolumn{4}{|l|}{ Current alcohol use, $n$ (\%) } \\
\hline Yes & 26 (38.8\%) & $14(40.0 \%)$ & 0.956 \\
\hline No & $41(61.2 \%)$ & $21(60.0 \%)$ & \\
\hline \multicolumn{4}{|l|}{ HIV-related characteristics } \\
\hline Time since HIV diagnosis, years, (median, IQR) & $5.2(3.8,8.2)$ & $4.8(3.2,7.5)$ & 0.782 \\
\hline Duration on cART, years (median, IQR) & $4.3(3.7,6.3)$ & $4.0(2.9,7.6)$ & 0.578 \\
\hline Sexual preference (homosexual, \%) & $14(20.9 \%)$ & $9(25.7 \%)$ & 0.554 \\
\hline Current CD4 count, cells/ $\mu \mathrm{l}($ mean $\pm \mathrm{SD})$ & $414.9 \pm 194.9$ & $472.2 \pm 230.5$ & 0.215 \\
\hline \multicolumn{4}{|l|}{ Clinical symptom } \\
\hline Hypertension & $32(47.7 \%)$ & $15(42.8 \%)$ & 0.572 \\
\hline History of diabetes & $6(9.0 \%)$ & $2(5.7 \%)$ & 0.569 \\
\hline Depressive symptoms & $27(40.3 \%)$ & $13(37.1 \%)$ & 0.759 \\
\hline Insomnia symptoms & $13(19.4 \%)$ & $5(14.3 \%)$ & 0.510 \\
\hline Frailty symptoms & $18(26.9 \%)$ & $10(28.6 \%)$ & 0.858 \\
\hline \multicolumn{4}{|l|}{ Laboratory biomarkers } \\
\hline HDL (median, IQR) & $1.2(0.9,1.5)$ & $1.2(1.0,1.5)$ & 0.736 \\
\hline LDL (median, IQR) & $2.3(1.8,2.9)$ & $2.5(2.1,3.0)$ & 0.401 \\
\hline CHOL (median, IQR) & $4.7(3.9,5.4)$ & $4.5(3.9,4.9)$ & 0.242 \\
\hline TG (median, IQR) & $2.0(1.2,3.2)$ & $1.7(1.0,3.0)$ & 0.371 \\
\hline Left CIMT (median, IQR) & $1.1(0.7,1.4)$ & $0.9(0.8,1.0)$ & $0.017^{\star}$ \\
\hline Right CIMT (median, IQR) & $1.2(0.8,1.2)$ & $0.9(0.8,1.0)$ & $0.008^{\star \star}$ \\
\hline \multicolumn{4}{|l|}{ Carotid plaques, $n(\%)$} \\
\hline Yes & 36 (53.7\%) & $9(25.7 \%)$ & $0.007^{\star \star}$ \\
\hline No & $31(46.3 \%)$ & $26(74.3 \%)$ & \\
\hline \multicolumn{4}{|l|}{ Cholesterol crystal, $n(\%)$} \\
\hline Yes & 20 (29.9\%) & 1 (2.9\%) & $0.001^{\star \star}$ \\
\hline No & 47 (70.1\%) & $34(52.2 \%)$ & \\
\hline
\end{tabular}

$\mathrm{NCl}$, neurocognitive impairment; BMI, body mass index; CIMT, carotid intima-media thickness; IQR, interquartile range; SD, standard deviation.

${ }^{*} P<0.05 ;{ }^{* *} P<0.01$.

Fourteen metabolites [bile acids (BAs), glycerophosphoinositols, fatty acids, eicosanoids, and fatty amides] were significantly increased in the NCI group compared to the non-NCI group. Four vitamin D metabolites [1alpha,25-dihydroxy-2alpha-(3-hydroxypropoxy)-19norvitamin D3, (20S)-24-hydroxy-19-norgeminivitamin D3, 24,24difluoro-1,25,26-trihydroxyvitamin D3, and atocalcitol], five terpenoids [astaxanthin diglucoside/astaxanthin $\beta$-D-diglucoside, lutein, tangeraxanthin, (-)-fusicoplagin $\mathrm{A}$, and 18alphahydroxyglycyrrhetic acid], and resolvin D1 were significantly decreased in the NCI group compared with the non-NCI group (Supplementary Table S6).

\section{Correlation of the Gut Microbiota and Fecal Metabolites}

Multiple correlations were found between fecal metabolites and specific gut bacteria (Figure 4). The metabolites which increased in the NCI group were consistently negatively correlated with Faecalibacterium, Corprococcus_2, and Ruminococcus_1 and positively correlated with Klebsiella. The metabolites which decreased in the NCI group, including four vitamin D metabolites, five terpenoids, and resolvin D1, were positively correlated with Faecalibacterium, Corprococcus_2, and Ruminococcus_1, whereas they were negatively correlated with Klebsiella.

\section{Correlation Between Plasma 25(OH)D With Clinical Parameters and Gut Microbiota}

We further measured the level of plasma 25(OH)D to verify our previous results. The median $25(\mathrm{OH}) \mathrm{D}$ level was $33.1 \mathrm{ng} / \mathrm{ml}$ in this population. The plasma $25(\mathrm{OH}) \mathrm{D}$ was deficient in one $(1 \%)$ participant, insufficient in 23 (22.5\%) participants, and normal in $78(76.5 \%)$ participants. The $25(\mathrm{OH}) \mathrm{D}$ level was significantly lower in the NCI group than that in the non-NCI group $(P<$ 0.001 ) (Figure 5). The plasma $25(\mathrm{OH}) \mathrm{D}$ was negatively associated with the left $(P=0.021)$ and the right CIMT $(P=$ $0.007)$. Furthermore, the plasma $25(\mathrm{OH}) \mathrm{D}$ was positively 

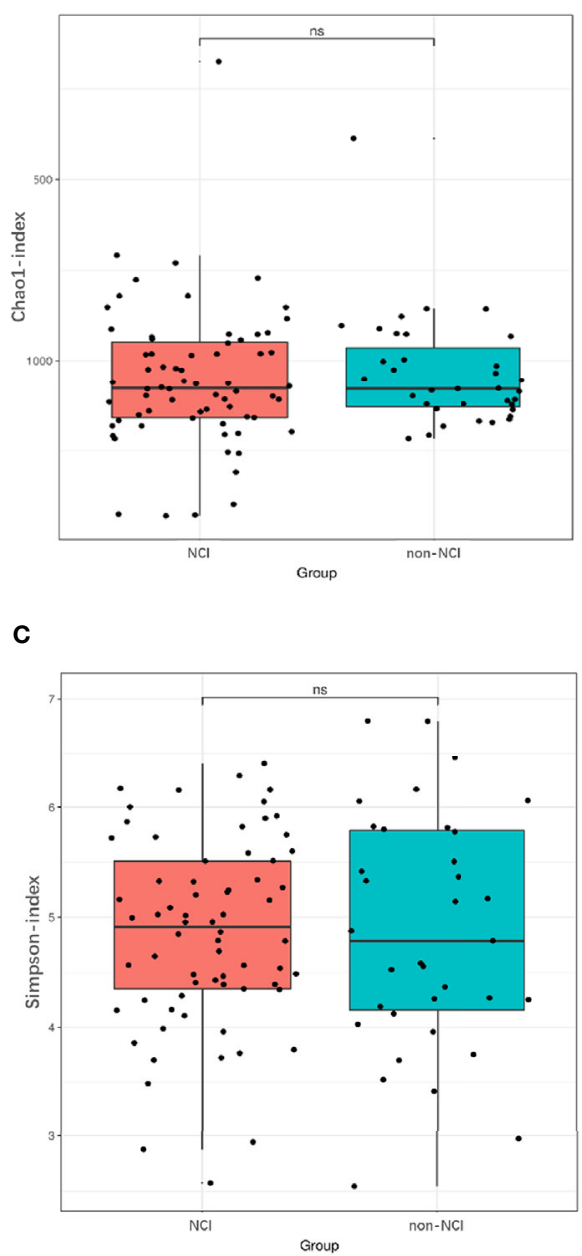

(
B

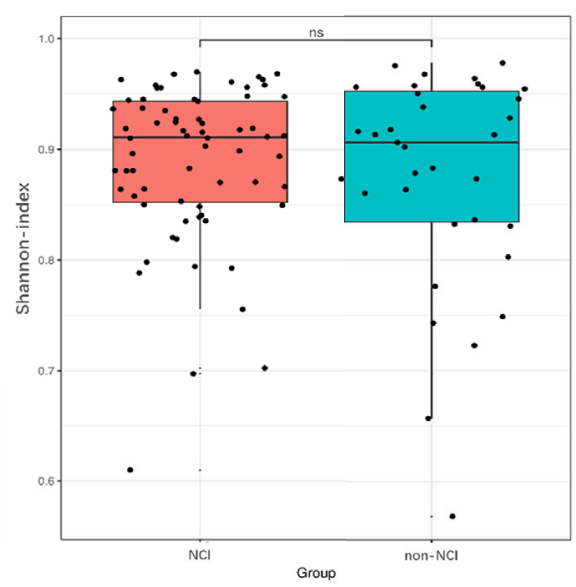

FIGURE 1 | Differences in $\alpha$-diversity of gut microbiota between $\mathrm{NCl}$ and non- $\mathrm{NCl}$ groups. NCl group, HIV-infected persons with neurocognitive impairment; non$\mathrm{NCl}, \mathrm{HIV}$-infected persons without neurocognitive impairment; ns, not significantly different $\alpha$-diversity of gut microbiota from the $\mathrm{NCl}$ group compared with the non$\mathrm{NCl}$ group. Statistical significance was set at $P=0.05$. (A) Chao1 index, (B) Shannon index, and (C) Simpson index.

associated with Faecalibacterium, Corprococcus_2, and Ruminococcaceae_NK4A214_groups but negatively associated with Ruminococcus_1, Eubacterium_eligens_group, and uncultured_bacterium (Table 2).

\section{DISCUSSION}

To the best of our knowledge, this is the first study using an integrated 16S rRNA gene sequencing and non-targeted fecal metabolomics approach to investigate the association of the gut microbiota and fecal metabolic phenotype with NCI in HIVinfected individuals. We found some significant differences between the NCI and non-NCI groups in terms of the abundance of gut microbiota at the genera level as well as in levels of several bacteria-related fecal metabolites. We also found significant correlations between the abundance of microbiota and fecal metabolites.

A number of studies suggested that gut microbiota dysbiosis in HIV-infected individuals is common (Ribeiro et al., 2017; Bandera et al., 2018). However, the role of gut microbiota dysbiosis in the pathogenesis of NCI in HIV-infected individuals is still an understudied area. We only identified one study from literature that showed a significantly lower a-diversity in participants with HAND compared to those without HAND in HIV-infected individuals. To exclude confounding bias, the authors matched the two groups for well-known confounders, including age, gender, education status, CD4 count, and sexual preference, that underwent further analysis. They found that there were no significant differences in a-diversity, $\beta$-diversity, and microbiota composition between the two groups (Zhang et al., 2019). In our study, the NCI and non-NCI groups also showed comparable a-diversity and $\beta$-diversity with comparable 


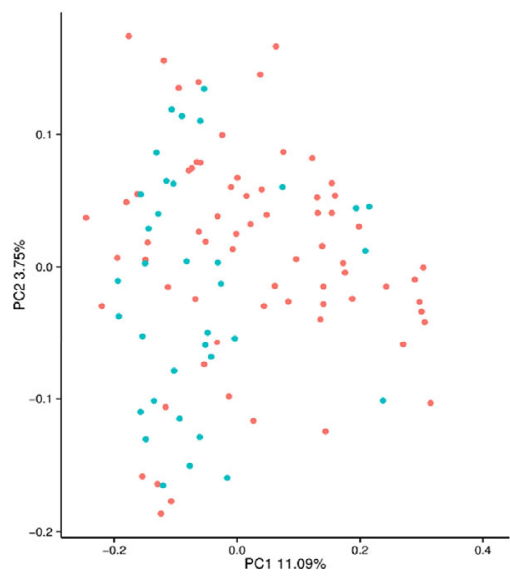

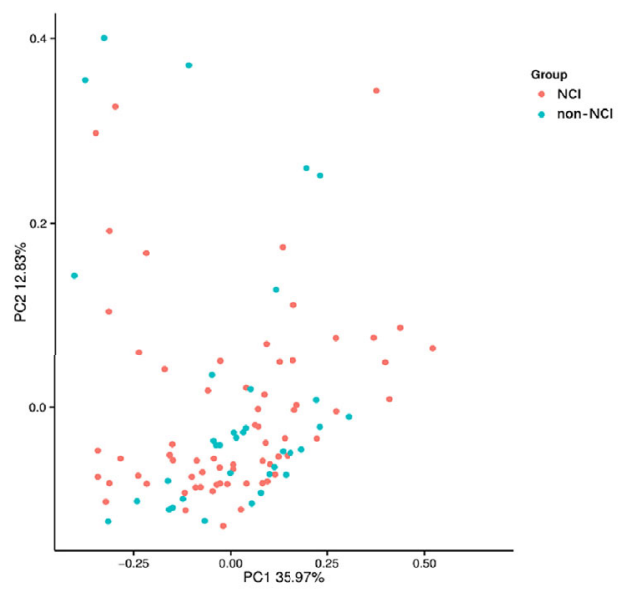

FIGURE 2 | Unweighted (A) and weighted (B) analyses of similarities and principal coordinate analysis based on the distance matrix of UniFrac dissimilarity of the fecal microbial communities in the $\mathrm{NCl}$ and non- $\mathrm{NCl}$ groups. $\mathrm{NCl}$ group, HIV-infected persons with neurocognitive impairment; non- $\mathrm{NCl}$, HIV-infected persons without neurocognitive impairment; PC, principal components. Each symbol represents a sample.

potential confounders, while some significant differences in gut composition were identified between the two groups.

Among the genera with observed differences between the NCI and non-NCI groups, Faecalibacterium, Corprococcus_2, Ruminococcaceae_NK4A214_group, and Ruminococcus_1 contain butyrate-producing bacteria (BPB). They were increased in the non-NCI group compared with the NCI group and were negatively correlated with CIMT. This result is supported by previously observed links between BPB and numerous neurocognitive disorders, including Parkinson's disease, Alzheimer's disease, and depression (Liu et al., 2020; Nuzum et al., 2020). It is now well established that BPB can provide energy for intestinal cells and to protect the gut barrier. The loss of BPB (Faecalibacterium or Coprococcus) could lead to a loss of integrity of the gut barrier and protection against intestinal epithelium inflammation (Liu et al., 2020; Nuzum et al., 2020). Our findings support that a low abundance of BPB was associated with NCI and may support a link between altered gut microbiota and the chronic, low-grade inflammation often observed in NCI patients. We also found that Klebsiella, which is an opportunistic pathogenic taxon, was increased in the NCI group and positively correlated with CIMT. Klebsiella has been found to be frequently distributed in hypertensive gut microbiome (Yan et al., 2017). Thus, BPB and Klebsiella may play a role in the potential pathogenesis behind NCI and carotid atherosclerosis.

We further studied the metabolic byproducts of intestinal bacteria in fecal samples. NCI appeared to be associated with several fecal metabolites. Consistent with previous studies, we found that specific BAs and bioactive lipids [glycerophospholipids, fatty acids, eicosanoids, and endocannabinoids (anandamide, 20:1, $n$ - 9)] may have a role in influencing the cognitive function (MahmoudianDehkordi et al., 2019; Baptista et al., 2020). Increasing evidence suggests that BAs could serve as biomarkers of cognitive aging and Alzheimer's disease (MahmoudianDehkordi et al., 2019; Nho et al., 2019). Recently, a significant increase in the levels of bacterially produced secondary BAs (deoxycholic acid) and their conjugated forms in $\mathrm{AD}$ patients was noted compared to those in cognitively normal older adults (MahmoudianDehkordi et al., 2019). Earlier studies also identified increased levels of several secondary BAs in mild cognitive impairment and $\mathrm{AD}$ patients compared with those of cognitively normal controls (Mapstone et al., 2014; Marksteiner et al., 2018). However, inconsistent with those previous findings, we observed increased levels of specific primary BAs (glycocholic acid and chenodeoxycholic acid glycine conjugate) in NCI patients compared to those in non-NCI subjects. The differences may be attributed to the different population. To our best knowledge, most of the previous studies were conducted in the general population. Thus, on the one hand, the links that we observed need to be tested in large populations. Moreover, additional experimental studies are needed to fully define the mechanistic roles of BAs in the development of NCI in HIVinfected patients.

In addition, we, for the first time, identified significant declines of multiple dietary and nutritional constituents known to exert anti-inflammatory and antioxidant properties, including vitamin $\mathrm{D}$, terpenoids, and resolvin $\mathrm{D} 1$ in the NCI group compared with the non-NCI group. Terpenoids may be considered useful modulators of brain-derived neurotrophic factor (BDNF) in CNS diseases-for example, in vitro studies have found that the BDNF expression activities were positively associated with the terpenoids (Sangiovanni et al., 2017; Gonulalan et al., 2020). For resolving D1, a study reported that the early chronic treatment of rats overexpressing human $\alpha$ synuclein with resolving D1 prevents central and peripheral inflammation as well as neuronal dysfunction and motor deficits. They also reported that central and peripheral resolving D1 is decreased in early-PD patients (Krashia et al., 


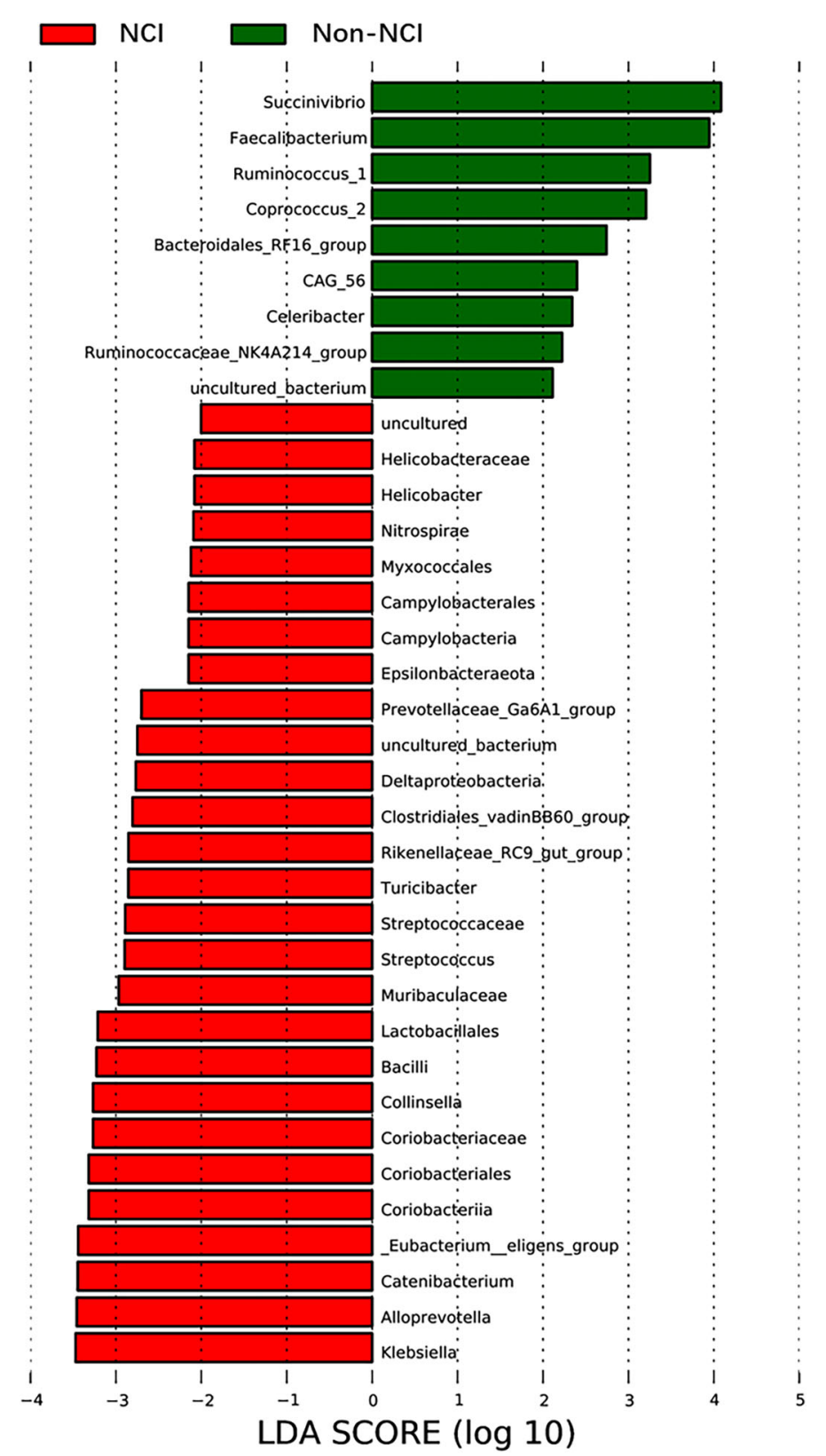

FIGURE 3 | LEfSe was used to identify the most differentially abundant taxa of bacterial communities between the NCl and non-NCl groups, with LDA score $>2$ (b, d). NCl group, HIV-infected persons with neurocognitive impairment; non- $\mathrm{NCl}$, HIV-infected persons without neurocognitive impairment; LEfSe, linear discriminant analysis effect size; LDA, linear discriminant analysis.

2019). However, human evidence is very limited. Further clinical studies are needed to confirm these constituents as diagnostic biomarkers and disease-modifying agents.

Vitamin D may be an important relevant dietary aspect in HIV-infected people for several reasons. First, previous studies reported that vitamin D insufficiency $[25(\mathrm{OH}) \mathrm{D}<30 \mathrm{ng} / \mathrm{ml}]$ is highly prevalent, up to $60-85 \%$ depending on demographic information, geographic information, and type of cART in HIV-1 infected populations (Manion et al., 2017; Mastaglia et al., 2017). Second, vitamin D insufficiency or deficiency has been associated with NCI, subclinical vascular disease, and several HIV disease outcomes (Vergori et al., 2019). In this study, the median plasma $25(\mathrm{OH}) \mathrm{D}$ was $33.1 \mathrm{ng} / \mathrm{ml}$, and the prevalence of vitamin D insufficiency was $22.5 \%$. Our study population had relatively better vitamin D status when compared with the previous data (Manion et al., 2017; Mastaglia et al., 2017), whereas we still found the association between vitamin D insufficiency and NCI. On one hand, vitamin D is the main subclass of fecal metabolites decreased in the NCI group compared with the non-NCI group. On the other hand, the 


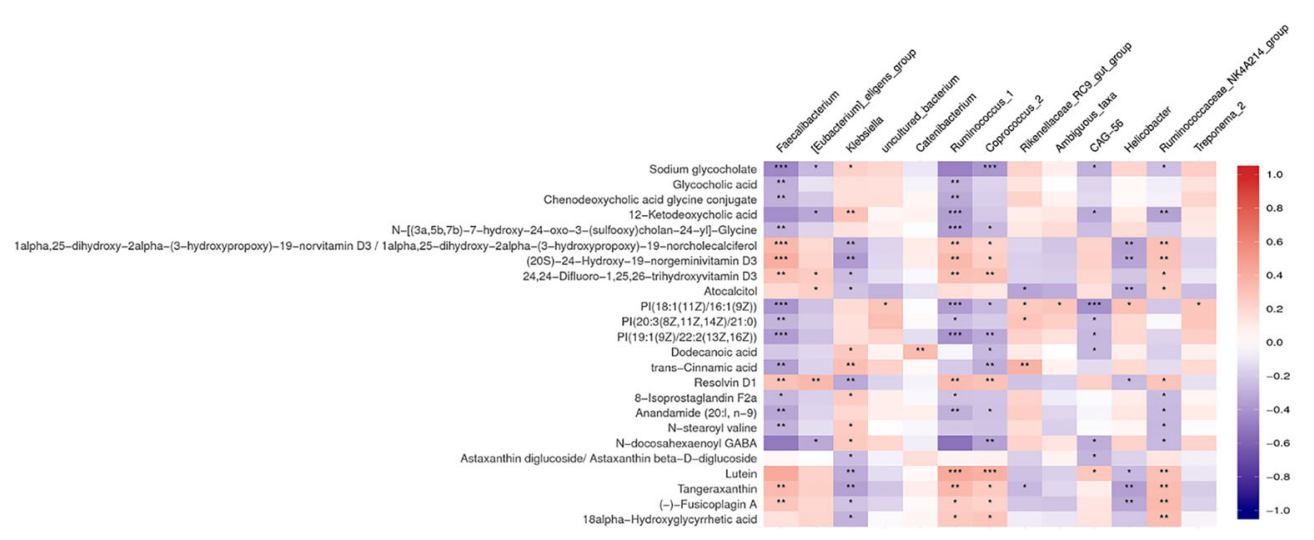

FIGURE 4 | Heat map summarizing the correlation of perturbed gut microbiota genera and altered fecal metabolites between the $\mathrm{NCl}$ and non- $\mathrm{NCl}$ groups. ${ }^{*} P<0.05$; ${ }^{\star \star} P<0.01 ;{ }^{* \star} P<0.001$.

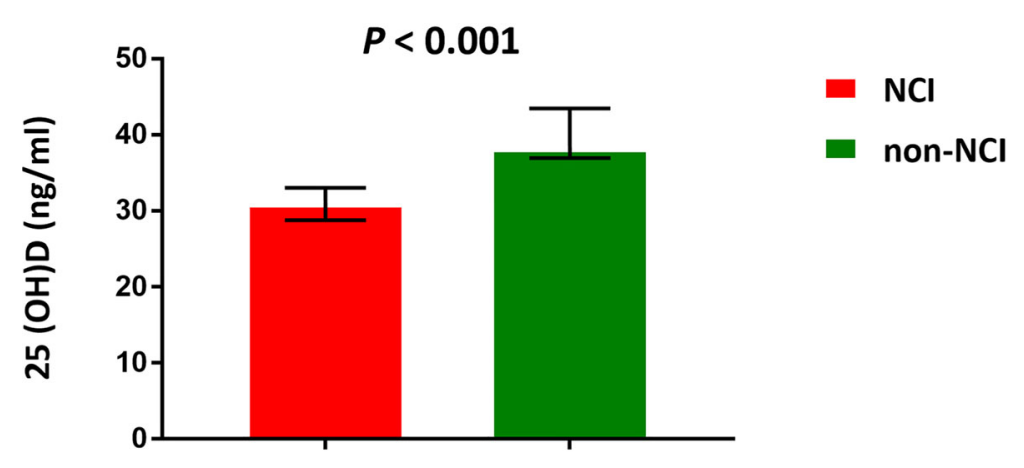

FIGURE 5 | Median concentrations of plasma 25(OH)D. NCl group, HIV-infected persons with neurocognitive impairment; non-NCl, HIV-infected persons without neurocognitive impairment. The error bars represent interquartile range.

plasma $25(\mathrm{OH}) \mathrm{D}$ in the NCI group was significantly lower than that in the non-NCI group. $25(\mathrm{OH}) \mathrm{D}$ was also negatively associated with the left and right CIMT. 25(OH)D has been associated to NCI and CIMT in many studies (Vergori et al., 2019; Zugic Soares et al., 2019; Gonzalez-Martin et al., 2020). Different mechanisms have been suggested to explain the neuroprotective effects of $25(\mathrm{OH}) \mathrm{D}$, including the regulation of inflammation, reactive oxygen species, and calcium homeostasis as well as vessel protection (Schlogl and Holick, 2014). It should be noted that we firstly found that fecal vitamin $\mathrm{D}$ metabolites were associated with NCI. There is little information in literature on fecal vitamin D level and health risk. To our knowledge, only one recent study found that vitamin D was significantly decreased in the feces of hypertension patients and positively correlated with hypertension-reduced bacterial genera (Bashir et al., 2016). It should be noted that regulation of the circulating

TABLE 2 | The correlation between 25(OH)D and the abundance of genera [left and right carotid intima-media thickness (CIMT)].

\begin{tabular}{|c|c|c|c|}
\hline & Correlation & $P$-value & Adjusted $P$-value \\
\hline Right CIMT & -0.319 & $0.001^{\star \star}$ & $0.007^{\star \star}$ \\
\hline Ruminococcus_1 & -0.289 & $0.003^{\star \star}$ & $0.014^{*}$ \\
\hline Coprococcus_2 & 0.335 & $<0.001^{\star \star \star}$ & $0.007^{\star \star}$ \\
\hline Ruminococcaceae_NK4A214_group & 0.188 & $0.049^{\star}$ & 0.101 \\
\hline Eubacterium._eligens_group & -0.245 & $0.013^{*}$ & $0.043^{*}$ \\
\hline
\end{tabular}

${ }^{*} P<0.05 ;{ }^{* *} P<0.01 ;{ }^{* * *} P<0.001$. 
levels of $25(\mathrm{OH}) \mathrm{D}$ depends mainly on sun exposure and nutritional intake. As an observational study, it remains unclear whether the decreased level of $25(\mathrm{OH}) \mathrm{D}$ in the NCI group (we observed differences) that we note causes or is a consequence of NCI. Therefore, we could not exclude the possibility of an inverse causal link.

In addition, we evaluated the association between gut microbiota perturbations and fecal metabolites. Herein we observed significant associations of BPB and Klebsiella with several fecal metabolites, including vitamin $\mathrm{D}$ metabolites. The metabolites with a higher level in the NCI group compared to the non-NCI group were negatively correlated with BPB and positively correlated with Klebsiella. The metabolites with a lower level in the NCI group were positively correlated with $\mathrm{BPB}$, whereas these were negatively correlated with Klebsiella. These findings suggest that these metabolites may mediate the associations of BPB and Klebsiella with NCI. Consistently, both fecal and plasma vitamin D were positively associated with BPB (Faecalibacterium, Corprococcus_2, and Ruminococcaceae_NK4A214_groups), while these were negatively associated with Ruminococcus_1. In agreement with our findings, some studies reported an increase in abundance of Coprococcus and Faecalibacterium and a decreased abundance of genus Ruminococcus after vitamin D supplementation (Bashir et al., 2016; Naderpoor et al., 2019). However, open-label pilot studies and randomized, double-blind trials examining the effect of vitamin $\mathrm{D}$ on human gut microbiota did not reach a conclusion. Further studies are needed to fully understand the relation between vitamin $\mathrm{D}$ and gut microbiota.

The present study has some limitations. First, the relatively small sample size might lead to a low statistical power. Second, the study design cannot prescribe a causal mechanism. Future studies are needed to elucidate the causal relationship.

\section{CONCLUSION}

$\mathrm{BPB}$, Klebsiella, and associated metabolites may play a role in the pathogenesis of NCI. In addition, this is the first time that we found vitamin $\mathrm{D}$, both in feces and blood, to be negatively associated with NCI and positively correlated with BPB.

\section{REFERENCES}

Ashuro, A. A., Lobie, T. A., Ye, D. Q., Leng, R. X., Li, B. Z., Pan, H. F., et al. (2020). Review on the Alteration of Gut Microbiota: The Role of HIV Infection and Old Age. AIDS Res. Hum. Retroviruses 36 (7), 556-565. doi: 10.1089/ aid.2019.0282

Bandera, A., De Benedetto, I., Bozzi, G., and Gori, A. (2018). Altered Gut Microbiome Composition in HIV Infection: Causes, Effects and Potential Intervention. Curr. Opin. HIV AIDS 13 (1), 73-80. doi: 10.1097/ COH.0000000000000429

Baptista, L. C., Sun, Y., Carter, C. S., and Buford, T. W. (2020). Crosstalk Between the Gut Microbiome and Bioactive Lipids: Therapeutic Targets in Cognitive Frailty. Front. Nutr. 7. doi: 10.3389/fnut.2020.00017

Bashir, M., Prietl, B., Tauschmann, M., Mautner, S. I., Kump, P. K., Treiber, G., et al. (2016). Effects of High Doses of Vitamin D3 on Mucosa-Associated Gut Microbiome Vary Between Regions of the Human Gastrointestinal Tract. Eur. J. Nutr. 55 (4), 1479-1489. doi: 10.1007/s00394-015-0966-2

\section{DATA AVAILABILITY STATEMENT}

The raw data supporting the conclusions of this article will be made available by the authors, without undue reservation.

\section{ETHICS STATEMENT}

The studies involving human participants were reviewed and approved by the Committee of the School of Public Health at Fudan University. The patients/participants provided their written informed consent to participate in this study.

\section{AUTHOR CONTRIBUTIONS}

$\mathrm{RD}$ and $\mathrm{NH}$ proposed and developed the research question. HL and $\mathrm{NH}$ generally supervised the study. RD, XC, RS, SY, JL, BZ, $\mathrm{XX}$, and KW contributed to data collection and data management. WS contributed to laboratory management and tests. RD performed data analysis. $\mathrm{RD}, \mathrm{NH}$, and XS wrote, reviewed, and edited the manuscript. DD provided expertise on neuropsychological tests. All authors contributed to the article and approved the submitted version.

\section{FUNDING}

This study was supported by the National Natural Science Foundation of China (81773485) and the China National Science and Technology Major Projects on Infectious Diseases (2018ZX10721102-004) and partially supported by the Shanghai Municipal Health Commission (GWV-10.1-XK16).

\section{SUPPLEMENTARY MATERIAL}

The Supplementary Material for this article can be found online at: https:/www.frontiersin.org/articles/10.3389/fcimb.2021.723840/ full\#supplementary-material

Cysique, L. A., Jin, H., Franklin, D. R.Jr., Morgan, E. E., Shi, C., Yu, X., et al. (2007). Neurobehavioral Effects of HIV-1 Infection in China and the United States: A Pilot Study. J. Int. Neuropsychol. Soc. 13 (5), 781-790. doi: 10.1017/ S1355617707071007

Ding, Y. Y., Lin, H. J., Liu, X., Wong, F. Y., Sun, Y. V., Marconi, V. C., et al. (2017a). Higher Prevalence of Frailty Among a Sample of HIV-Infected Middle-Aged and Older Chinese Adults Is Associated With Neurocognitive Impairment and Depressive Symptoms. J. Infect. Dis. 215 (5), 687-692. doi: 10.1093/infdis/jix032

Ding, Y. Y., Lin, H. J., Shen, W. W., Wu, Q. H., Gao, M. Y., and He, N. (2017b). Interaction Effects Between HIV and Aging on Selective Neurocognitive Impairment. J. Neuroimmune Pharmacol. 12 (4), 661-669. doi: 10.1007/ s11481-017-9748-3

Ding, Y., Zhu, B., Lin, H., Chen, X., Shen, W., Xu, X., et al. (2020). HIV Infection and Electrocardiogram Abnormalities: Baseline Assessment From the CHART Cohort. Clin. Microbiol. Infect. 26 (12), 1689.e1-1689.e7. doi: 10.1016/ j.cmi.2020.03.005 
Gonulalan, E. M., Nemutlu, E., Bayazeid, O., Kocak, E., Yalcin, F. N., and Demirezer, L. O. (2020). Metabolomics and Proteomics Profiles of Some Medicinal Plants and Correlation With BDNF Activity. Phytomedicine 74, 152920. doi: 10.1016/j.phymed.2019.152920

Gonzalez-Martin, J. J., Novella-Navarro, M., Calvo-Aranda, E., Cabrera-Alarcon, J. L., Carrion, O., Abdelkader, A., et al. (2020). Endothelial Dysfunction and Subclinical Atheromatosis in Patients With Systemic Sclerosis. Clin. Exp. Rheumatol 38 Suppl 125 (3), 48-52.

Heaton, R. K., Clifford, D. B., Franklin, D. R. Jr., Woods, S. P., Ake, C., Vaida, F., et al. (2010). HIV-Associated Neurocognitive Disorders Persist in the Era of Potent Antiretroviral Therapy: CHARTER Study. Neurology 75 (23), 20872096. doi: 10.1212/WNL.0b013e318200d727

Jenkins, C. D., Stanton, B. A., Niemcryk, S. J., and Rose, R. M. (1988). A Scale for the Estimation of Sleep Problems in Clinical Research. J. Clin. Epidemiol. 41 (4), 313-321. doi: 10.1016/0895-4356(88)90138-2

Ji, Y. J., Zhang, F. D., Zhang, R. F., Shen, Y. Z., Liu, L., Wang, J. R., et al. (2018). Changes in Intestinal Microbiota in HIV-1-Infected Subjects Following cART Initiation: Influence of CD4+T Cell Count. Emerging Microbes Infect. 7 (1), 113. doi: 10.1038/s41426-018-0117-y

Krashia, P., Cordella, A., Nobili, A., La Barbera, L., Federici, M., Leuti, A., et al. (2019). Blunting Neuroinflammation With Resolvin D1 Prevents Early Pathology in a Rat Model of Parkinson's Disease. Nat. Commun. 10 (1), 3945. doi: 10.1038/s41467-019-11928-w

Li, B. Y., He, Y. X., Ma, J. F., Huang, P., Du, J. J., Cao, L., et al. (2019). Mild Cognitive Impairment has Similar Alterations as Alzheimer's Disease in Gut Microbiota. Alzheimers Dementia 15 (10), 1357-1366. doi: 10.1016/ j.jalz.2019.07.002

Liu, R. T., Rowan-Nash, A. D., Sheehan, A. E., Walsh, R. F. L., Sanzari, C. M., Korry, B. J., et al. (2020). Reductions in Anti-Inflammatory Gut Bacteria are Associated With Depression in a Sample of Young Adults. Brain Behav. Immun. 88, 308-324. doi: 10.1016/j.bbi.2020.03.026

Luan, H. M., Wang, X., and Cai, Z. W. (2019). Mass Spectrometry-Based Metabolomics: Targeting the Crosstalk Between Gut Microbiota and Brain in Neurodegenerative Disorders. Mass Spectrometry Rev. 38 (1), 22-33. doi: $10.1002 /$ mas. 21553

MahmoudianDehkordi, S., Arnold, M., Nho, K., Ahmad, S., Jia, W., Xie, G., et al. (2019). Altered Bile Acid Profile Associates With Cognitive Impairment in Alzheimer's Disease-An Emerging Role for Gut Microbiome. Alzheimers Dement 15 (1), 76-92. doi: 10.1016/j.jalz.2018.07.217

Manion, M., Hullsiek, K. H., Wilson, E. M. P., Rhame, F., Kojic, E., Gibson, D., et al. (2017). Vitamin D Deficiency is Associated With IL-6 Levels and Monocyte Activation in HIV-Infected Persons. PloS One 12 (5), e0175517. doi: 10.1371/journal.pone.0175517

Mapstone, M., Cheema, A. K., Fiandaca, M. S., Zhong, X. G., Mhyre, T. R., MacArthur, L. H., et al. (2014). Plasma Phospholipids Identify Antecedent Memory Impairment in Older Adults. Nat. Med. 20 (4), 415-41+. doi: 10.1038/nm.3466

Marksteiner, J., Blasko, I., Kemmler, G., Koal, T., and Humpel, C. (2018). Bile Acid Quantification of 20 Plasma Metabolites Identifies Lithocholic Acid as a Putative Biomarker in Alzheimer's Disease. Metabolomics 14 (1), 1. doi: 10.1007/s11306-017-1297-5

Mastaglia, S., Watson, D., Bello, N., Fridman, V., Stecher, D., and Oliveri, B. (2017). Vitamin D Levels and Their Impact on Mineral Metabolism in HIV Infected Patients: An Exploratory Study. Clin. Cases Miner Bone Metab. 14 (1), 18-22. doi: 10.11138/ccmbm/2017.14.1.018

Naderpoor, N., Mousa, A., Fernanda Gomez Arango, L., Barrett, H. L., Dekker Nitert, M., and de Courten, B. (2019). Effect of Vitamin D Supplementation on Faecal Microbiota: A Randomised Clinical Trial. Nutrients 11 (12), 2888. doi: 10.3390/nu11122888

Nho, K., Kueider-Paisley, A., MahmoudianDehkordi, S., Arnold, M., Risacher, S. L., Louie, G., et al. (2019). Altered Bile Acid Profile in Mild Cognitive Impairment and Alzheimer's Disease: Relationship to Neuroimaging and CSF Biomarkers. Alzheimers Dement 15 (2), 232-244. doi: 10.1016/j.jalz.2018.08.012

Nuzum, N. D., Loughman, A., Szymlek-Gay, E. A., Hendy, A., Teo, W. P., and Macpherson, H. (2020). Gut Microbiota Differences Between Healthy Older Adults and Individuals With Parkinson's Disease: A Systematic Review. Neurosci. Biobehav. Rev. 112, 227-241. doi: 10.1016/j.neubiorev.2020.02.003
Qiao, X. T., Lin, H. J., Chen, X. X., Ning, C. X., Wang, K. R., Shen, W. W., et al. (2019). Sex Differences in Neurocognitive Screening Among Adults Living With HIV in China. J. Neurovirology 25 (3), 363-371. doi: 10.1007/s13365019-00727-0

Ribeiro, A., Heimesaat, M. M., and Bereswill, S. (2017). Changes of the Intestinal Microbiome-Host Homeostasis in HIV-Infected Individuals - A Focus on the Bacterial Gut Microbiome. Eur. J. Microbiol. Immunol. (Bp) 7 (3), 158-167. doi: 10.1556/1886.2017.00016

Sangiovanni, E., Brivio, P., Dell'Agli, M., and Calabrese, F. (2017). Botanicals as Modulators of Neuroplasticity: Focus on BDNF. Neural Plasticity 2017, 5965371. doi: 10.1155/2017/5965371

Schlogl, M., and Holick, M. F. (2014). Vitamin D and Neurocognitive Function. Clin. Interv Aging 9, 559-568. doi: 10.2147/CIA.S51785

Sun, Y., Ma, Y. F., Lin, P., Tang, Y. W., Yang, L. Y., Shen, Y. Z., et al. (2016). Fecal Bacterial Microbiome Diversity in Chronic HIV-Infected Patients in China. Emerging Microbes Infections 5 (4), e31. doi: 10.1038/emi.2016.25

Vergori, A., Pinnetti, C., Lorenzini, P., Brita, A., Libertone, R., Mastrorosa, I., et al. (2019). Vitamin D Deficiency is Associated With Neurocognitive Impairment in HIV-Infected Subjects. Infection 47 (6), 929-935. doi: 10.1007/s15010-01901313-6

Wan, Y., Wang, F. L., Yuan, J. H., Li, J., Jiang, D. D., Zhang, J. J., et al. (2019). Effects of Dietary Fat on Gut Microbiota and Faecal Metabolites, and Their Relationship With Cardiometabolic Risk Factors: A 6-Month Randomised Controlled-Feeding Trial. Gut 68 (8), 1417-141+. doi: 10.1136/gutjnl-2018317609

Williams, M. E., Ipser, J. C., Stein, D. J., Joska, J. A., and Naude, P. J. W. (2020). Peripheral Immune Dysregulation in the ART Era of HIV-Associated Neurocognitive Impairments: A Systematic Review. Psychoneuroendocrinology 118, 104689. doi: 10.1016/j.psyneuen.2020.104689

Yan, Q., Gu, Y., Li, X., Yang, W., Jia, L., Chen, C., et al. (2017). Alterations of the Gut Microbiome in Hypertension. Front. Cell Infect. Microbiol. 7, 381. doi: 10.3389/fcimb.2017.00381

Yu, M., Jia, H., Zhou, C., Yang, Y., Zhao, Y., Yang, M., et al. (2017). Variations in Gut Microbiota and Fecal Metabolic Phenotype Associated With Depression by $16 \mathrm{~S}$ rRNA Gene Sequencing and LC/MS-Based Metabolomics. J. Pharm. BioMed. Anal. 138, 231-239. doi: 10.1016/j.jpba.2017.02.008

Zhang, F. D., Yang, J. Y., Ji, Y. J., Sun, M. Y., Shen, J. Y., Sun, J. J., et al. (2019). Gut Microbiota Dysbiosis Is Not Independently Associated With Neurocognitive Impairment in People Living With HIV. Front. Microbiol. 9. doi: 10.3389/ fmicb.2018.03352

Zugic Soares, J., Pettersen, R., Saltyte Benth, J., Knapskog, A. B., Selbaek, G., and Bogdanovic, N. (2019). Higher Vitamin D Levels Are Associated With Better Attentional Functions: Data From the NorCog Register. J. Nutr. Health Aging 23 (8), 725-731. doi: 10.1007/s12603-019-1220-Z

Zung, W. W. (1973). From Art to Science. The Diagnosis and Treatment of Depression. Arch. Gen. Psychiatry 29 (3), 328-337. doi: 10.1001/ archpsyc.1973.04200030026004

Conflict of Interest: The authors declare that the research was conducted in the absence of any commercial or financial relationships that could be construed as a potential conflict of interest.

Publisher's Note: All claims expressed in this article are solely those of the authors and do not necessarily represent those of their affiliated organizations, or those of the publisher, the editors and the reviewers. Any product that may be evaluated in this article, or claim that may be made by its manufacturer, is not guaranteed or endorsed by the publisher.

Copyright (C) 2021 Dong, Lin, Chen, Shi, Yuan, Li, Zhu, Xu, Shen, Wang, Shu, Ding and He. This is an open-access article distributed under the terms of the Creative Commons Attribution License (CC BY). The use, distribution or reproduction in other forums is permitted, provided the original author(s) and the copyright owner(s) are credited and that the original publication in this journal is cited, in accordance with accepted academic practice. No use, distribution or reproduction is permitted which does not comply with these terms. 